\title{
A GCD problem and a Hessenberg determinant
}

\author{
M. Hariprasad \\ Department of Computational and Data Sciences \\ Indian Institute of Science, Bangalore-560012, India \\ e-mail: mhariprasadkansuregmail.com
}

Received: 31 December 2016

Accepted: 7 May 2018

\begin{abstract}
In this article we give a proof that, when two integers $a$ and $b$ are coprime $((a, b)=1$ i.e. greatest common divisor (GCD) of $a$ and $b$ is 1$)$, then GCD of $a+b$ and $\frac{a^{p}+b^{p}}{a+b}$ is either 1 or $p$ for a prime number $p$. We prove this by linking the problem to a certain type of Hessenberg determinants.
\end{abstract}

Keywords: Greatest common divisor, Binomial coefficients, Hessenberg determinants.

2010 Mathematics Subject Classification: 11A05, 15B36, 11C20.

\section{Introduction}

For integers $a$ and $b$ let $(a, b)$ denote the greatest common divisor (GCD). The book [1] has an exercise: If $(a, b)=1$ then prove that $\left(a+b, a^{2}-a b+b^{2}\right)$ is either 1 or 3 . Here we prove a generalized version of this problem by linking it to linear algebra. We prove that if $(a, b)=1$, then $\left(a+b, \frac{a^{p}+b^{p}}{a+b}\right)$ is either 1 or factors of $p$ when $p$ is an odd number. When $p$ is a prime number, then $\left(a+b, \frac{a^{p}+b^{p}}{a+b}\right)$ is either 1 or $p$.

\section{Results}

Lemma 2.1. If $(a, b)=1$, and some $d>1$ is such that $d \mid(a+b)$ then $d \nmid a$ and $d \nmid b$.

Proof. Suppose $d \mid a$ then $d \mid(a+b-a)$, which is $d \mid(b)$. But $(a, b)=1$ and $d>1$. By contradiction $d$ will not divide $a$. Similarly for $b$. 
Lemma 2.2. For an odd integer $p$,

$$
\sum_{n=1}^{(p-1) / 2}(-1)^{n-1}(p-2 n)\left(\begin{array}{l}
p \\
n
\end{array}\right)=p
$$

Proof. For a real $x>0$, consider $\left(x-\frac{1}{x}\right)^{p}$. By expanding this with binomial theorem we get

$$
\begin{aligned}
& \left(x-\frac{1}{x}\right)^{p}=\sum_{n=0}^{p}(-1)^{n}\left(\begin{array}{l}
p \\
n
\end{array}\right) x^{p-n}\left(\frac{1}{x}\right)^{n}, \\
& \left(x-\frac{1}{x}\right)^{p}=\sum_{n=0}^{p}(-1)^{n}\left(\begin{array}{l}
p \\
n
\end{array}\right) x^{p-2 n} .
\end{aligned}
$$

Differentiating equation 1 with respect to $x$,

$$
p\left(x-\frac{1}{x}\right)^{p-1}\left(1+\frac{1}{x^{2}}\right)=\sum_{n=0}^{p}(-1)^{n}\left(\begin{array}{l}
p \\
n
\end{array}\right)(p-2 n) x^{p-2 n-1} .
$$

Substitute $x=1$ in equation 3 , we get

$$
\begin{aligned}
& 0=2\left(\sum_{n=0}^{(p-1) / 2}(-1)^{n}\left(\begin{array}{l}
p \\
n
\end{array}\right)(p-2 n)\right), \\
& p=\sum_{n=1}^{(p-1) / 2}(-1)^{n-1}\left(\begin{array}{l}
p \\
n
\end{array}\right)(p-2 n) .
\end{aligned}
$$

Consider the lower Hessenberg matrices,

$$
H_{n}=\left[\begin{array}{cccccc}
\left(\begin{array}{c}
2 n+1 \\
1
\end{array}\right) & 1 & 0 & 0 & \cdots & 0 \\
\left(\begin{array}{c}
2 n+1 \\
2
\end{array}\right) & \left(\begin{array}{c}
2 n-1 \\
1
\end{array}\right) & 1 & 0 & \cdots & 0 \\
\left(\begin{array}{c}
2 n+1 \\
3
\end{array}\right) & \left(\begin{array}{c}
2 n-1 \\
2
\end{array}\right) & \left(\begin{array}{c}
2 n-3 \\
1
\end{array}\right) & 1 & \cdots & 0 \\
\vdots & \vdots & \cdots & \ddots & \ddots & 0 \\
\left(\begin{array}{c}
2 n+1 \\
n-1
\end{array}\right) & \left(\begin{array}{c}
2 n-1 \\
n-2
\end{array}\right) & \left(\begin{array}{c}
2 n-3 \\
n-3
\end{array}\right) & \cdots & 5 & 1 \\
\left(\begin{array}{c}
2 n+1 \\
n
\end{array}\right) & \left(\begin{array}{c}
2 n-1 \\
n-1
\end{array}\right) & \left(\begin{array}{c}
2 n-3 \\
n-2
\end{array}\right) & \cdots & 10 & 3
\end{array}\right] .
$$

With $H_{0}=1$ and $H_{1}=[3]$ and $H_{2}=\left[\begin{array}{cc}5 & 1 \\ 10 & 3\end{array}\right]$, etc.

Lemma 2.3. Determinant of the matrix $H_{n}$ is $2 n+1$.

Proof. We can see $\operatorname{det}\left(H_{1}\right)=3$ and $\operatorname{det}\left(H_{2}\right)=5$. Now by using principle of strong induction and expanding the determinant along the first row of $H_{n}$ we get the identity in Lemma 2.2 which proves Lemma 2.3.

Theorem 2.4. If $(a, b)=1$ then for an odd number $p=2 n+1,\left(a+b, \frac{a^{p}+b^{p}}{a+b}\right)=d$, where $d$ is $a$ divisor of $p$. 
Proof. Suppose $d \mid(a+b)$ and $d \mid \frac{a^{p}+b^{p}}{a+b}$ then we know that $d$ divides any linear combination of $(a+b)^{k}$ and $\frac{a^{p}+b^{p}}{a+b}$. We prove that this linear combination can give $p(a b)^{\frac{p-1}{2}}$. Then from Lemma 2.1 we deduce $d \mid p$. Let us suppose $a^{p}+b^{p}$ can be expressed in terms of $(a+b)^{k}$ for $k=$ $1,3,5, \cdots p$. So

$$
a^{p}+b^{p}=\sum_{k=0}^{(p-1) / 2} C_{k}(a+b)^{2 k+1} a^{\frac{p-1}{2}-k} b^{\frac{p-1}{2}-k}
$$

The equation 6 represents the linear combination of different powers of $(a+b)$. If we write this equation into the matrix form by considering as the first row as coefficient of $a^{p}$, second row as coefficients of $a^{p-1} b$, similarly $k^{\text {th }}$ row as coefficients of $a^{p-k} b^{k}$ and row $(p+1)$ having coefficients for $b^{p}$. We get the system,

$$
\left[\begin{array}{ccccccc}
1 & 0 & 0 & 0 & \cdots & 0 & 0 \\
\left(\begin{array}{c}
2 n+1 \\
1
\end{array}\right) & 1 & 0 & 0 & \cdots & 0 & 0 \\
\left(\begin{array}{c}
2 n+1 \\
2
\end{array}\right) & \left(\begin{array}{c}
2 n-1 \\
1
\end{array}\right) & 1 & 0 & \cdots & 0 & 0 \\
\left(\begin{array}{c}
2 n+1 \\
3
\end{array}\right) & \left(\begin{array}{c}
2 n-1 \\
2
\end{array}\right) & \left(\begin{array}{c}
2 n-3 \\
1
\end{array}\right) & 1 & \cdots & 0 & 0 \\
\vdots & \vdots & \cdots & \ddots & \ddots & 0 & 0 \\
\left(\begin{array}{c}
2 n+1 \\
n-1
\end{array}\right) & \left(\begin{array}{c}
2 n-1 \\
n-2
\end{array}\right) & \left(\begin{array}{c}
2 n-3 \\
n-3
\end{array}\right) & \left(\begin{array}{c}
2 n-5 \\
n-4
\end{array}\right) & \cdots & 1 & 0 \\
\left(\begin{array}{c}
2 n+1 \\
n
\end{array}\right) & \left(\begin{array}{c}
2 n-1 \\
n-1
\end{array}\right) & \left(\begin{array}{c}
2 n-3 \\
n-2
\end{array}\right) & \left(\begin{array}{c}
2 n-5 \\
n-3
\end{array}\right) & \cdots & 3 & 1 \\
\left(\begin{array}{c}
2 n+1 \\
n
\end{array}\right) & \left(\begin{array}{c}
2 n-1 \\
n-1
\end{array}\right) & \left(\begin{array}{c}
2 n-3 \\
n-2
\end{array}\right) & \left(\begin{array}{c}
2 n-5 \\
n-3
\end{array}\right) & \cdots & 3 & 1 \\
\left(\begin{array}{c}
2 n+1 \\
n-1
\end{array}\right) & \left(\begin{array}{c}
2 n-1 \\
n-2
\end{array}\right) & \left(\begin{array}{c}
2 n-3 \\
n-3
\end{array}\right) & \left(\begin{array}{c}
2 n-5 \\
n-4
\end{array}\right) & \cdots & 1 & 0 \\
\vdots & \vdots & \cdots & . \cdot & \cdot \cdot & 0 & 0 \\
\left(\begin{array}{c}
2 n+1 \\
3
\end{array}\right) & \left(\begin{array}{c}
2 n-1 \\
2
\end{array}\right) & \left(\begin{array}{c}
2 n-3 \\
1
\end{array}\right) & 1 & \cdots & 0 & 0 \\
\left(\begin{array}{c}
2 n+1 \\
2
\end{array}\right) & \left(\begin{array}{c}
2 n-1 \\
1
\end{array}\right) & 1 & 0 & \cdots & 0 & 0 \\
\left(\begin{array}{c}
2 n+1 \\
1
\end{array}\right) & 1 & 0 & 0 & \cdots & 0 & 0 \\
1 & 0 & 0 & 0 & \cdots & 0 & 0
\end{array}\right]\left[\begin{array}{c}
1 \\
c_{0} \\
c_{1} \\
c_{2} \\
c_{3} \\
c_{4} \\
\vdots \\
0 \\
c_{(p-1) / 2}
\end{array}\right]=\left[\begin{array}{c} 
\\
0 \\
\vdots \\
\vdots \\
\vdots \\
\vdots \\
\vdots \\
0 \\
0 \\
0 \\
1
\end{array}\right]
$$

Now because of the symmetry in binomial coefficients we can consider only the upper part of the matrix,

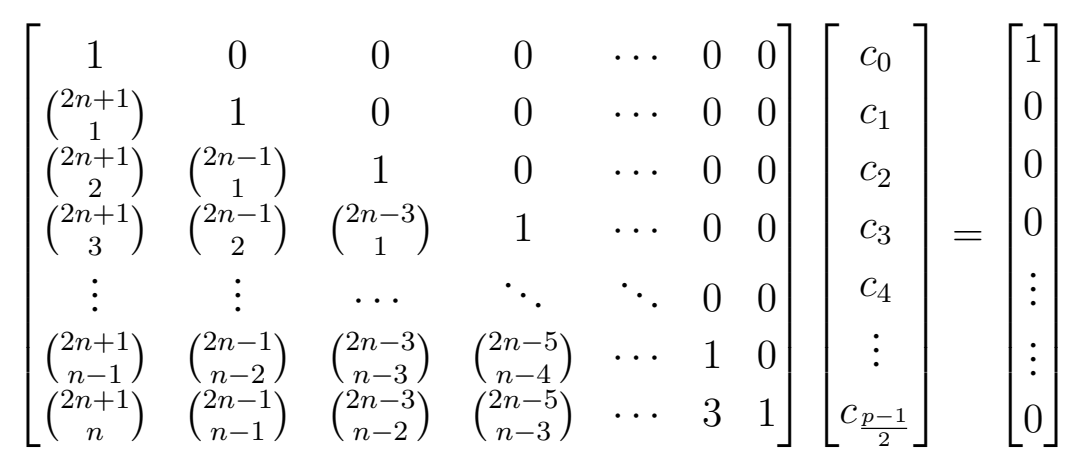

The equation (8) can be written as

$$
L x=b
$$

We need to find the $\frac{c_{\frac{p-1}{2}}}{2}$ which is the $((p+1) / 2,1)$ element in the matrix $L^{-1}$. Note that determinant of $L$ is 1 . 
And $(-1)^{(p-1) / 2} \operatorname{det}\left(H_{(p-1) / 2}\right)$ is the corresponding $((p+1) / 2,1)$ entry of $L^{-1}$. This is the determinant obtained by removing the first row and last column of the matrix $L$. From Lemma 2.3 it is nothing but $\pm p$.

Then from equation (6) we get

$$
p(a b)^{\frac{p-1}{2}}= \pm\left(\frac{a^{p}+b^{p}}{a+b}-\sum_{k=0}^{((p-1) / 2)-1} C_{k}(a+b)^{2 k}\right)
$$

$d$ divides RHS of equation (10), so it divides LHS, which proves the theorem.

\section{References}

[1] Apostol, T. M. (2013) Introduction to Analytic Number Theory, Springer Science \& Business Media. 